\title{
XXXVI. Krystallographische Untersuchung einiger organischer Verbindungen.
}

\author{
Von
}

P. Friedländer in Múnchen.

(Mit 6 Holzschnitten.)

\section{Diäthylphtalylketon.}

$$
\mathrm{C}_{8} \mathrm{H}_{4} \mathrm{O}_{2}\left(\mathrm{C}_{2} \mathrm{H}_{5}\right)_{2} \text {. }
$$

(Krystalle von Herrn Prof. V. M e yer in Zürich.)

Fig. 1.

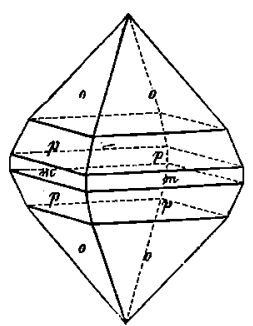

Krystallsystem quadratis ch.

$$
a: b=1: 1,0058 \text {. }
$$

Compacte, bis $1 \mathrm{~cm}$ dicke, durchsichtige Krystalle, durch Vorherrschen von o(111) meist oktaëdrisch entwickelt. Beobachtet wurden folgende Flächen (siehe Figur 1): $0=(111) P, p=(221) 2 P$, $m=(110) \infty P$.

Beobachtet: Berechnet:

$$
\begin{array}{lll}
o: 0=(111)(1 T 1)={ }^{*} 70^{0} 41^{\prime} & - \\
o: o=(111)(11 \overline{1}) & 7023 & 70^{\circ} 13^{\prime} \\
p: p=(221)(221) & 8329 & 8341 \\
m: p=(110)(221) & 1942 & 1922 \frac{1}{2} \\
o: p=(111)(221) & 1548 & 1553 \frac{1}{2}
\end{array}
$$

Eine senkrecht zur Hauptaxe geschliffene Platte zeigte im Polarisationsapparat das Interferenzbild einaxiger Krystalle. Doppelbrechung stark, positiv. Spaltbarkeit konnte an den Krystallen nicht wahrgenommen werden. 


\section{Diphenylenglycolsäureäthyläther.}

$\mathrm{C}_{14} \mathrm{H}_{9} \mathrm{O}_{3}, \mathrm{C}_{2} \mathrm{H}_{5}$. Schmelzpunkt $92^{0}$.

(P. Fried Iänder, Ber. d. d. chem. Ges. 1877, 10, 534.)

Krystallsystem a symmetrisch.

$$
a: b: c=1,0094: 1: 0,7922 .
$$

Axenwinkel im oberen rechten Oktanten

$$
\begin{array}{ll}
\alpha=90^{\circ} 36^{\prime} & A=89^{\circ} 53^{\prime} \\
\beta=11420 & B=11420 \\
\gamma=88 \quad 16 & C=8822
\end{array}
$$

Beobachtet wurden die Flächen: $a=(100) \infty \breve{P} \infty$, $b=(010) \infty \bar{P} \infty, c=(001) 0 P, m=(110) \infty P, p=(11 T) P$, $x=(21 \bar{T}) 2 \breve{P}, 2$ (s. Fig. 2). Durch sehr verschiedene Ausbildung der Flächen zeigen die Krystalle einen mannigfachen Habitus. Vorwiegend entwickelt ist meist $a(100)$, $b(010)$ und $c(001)$.

\begin{tabular}{|c|c|c|c|c|}
\hline$b: c=(010)(001)$ & $=* 900$ & $7^{\prime}$ & - & \\
\hline$a: c=(100)(001)$ & ${ }^{*} 65$ & 40 & - & \\
\hline$a: b=(100)(010)$ & $* 91$ & 38 & - & \\
\hline$m: b=(110)(010)$ & ${ }^{*} 48$ & 16 & & \\
\hline$p: c=(11 \pi)(00 \bar{T})$ & *55 & 54 & & \\
\hline$m: c=(110)(001)$ & 72 & $9 \frac{1}{2}$ & & $56^{\prime}$ \\
\hline$p: a=(11 T)(100)$ & 73 & 2 & 73 & 4 \\
\hline$x: a=(21 \overline{1})(100)$ & 45 & 35 & 45 & $7 \frac{1}{2}$ \\
\hline$x: c=(21 T)(00 \bar{T})$ & 77 & 29 & 77 & $39 \frac{1}{2}$ \\
\hline$x: p=(21 \pi)(11 \pi)$ & 27 & 26 & 27 & $56 \frac{1}{2}$ \\
\hline$m: c=(110)(11 \pi)$ & 51 & 57 & 52 & 10 \\
\hline
\end{tabular}

Fig. 2.

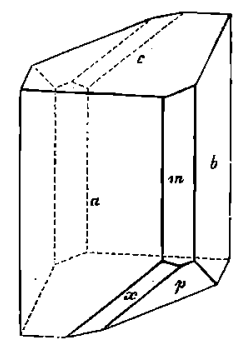

Beobachtet: Berechnet:

Weder durch $a(100)$, noch durcb $c$ sind optische Axen sichtbar. Die Auslöschungsrichtungen sind auf beiden Flächen gegen die Combinationskante geneigt. Spaltbarkeit nicht vorhanden.

\section{Cinchen.}

$$
\mathrm{C}_{19} \mathrm{H}_{20} \mathrm{~N}_{2} \text {. }
$$

(W. Königs, Ber. d. d. chem. Ges. 1881, 14, 1854.)

Krystallsystem rh o m b i s c h.

$$
a: b: c=0,6028: 1: 0,4972 .
$$

Die untersuchten Krystalle bilden kleine, dünne, nach $b=(010)$ entwickelte Täfelchen, an welchen als äusserst schmale Abstumpfungen der 
Kanten und zweier Ecken die Flächen o(111) und $m(110)$ beobachtet wurden (s. Fig. 3). Als Controlwinkel diente der ebene Winkel auf $b(010)$, welcher sich aus den gemessenen Kantenwinkeln zu $79^{\circ} 2^{\prime}$

Fig. 3. berechnet und $79^{\circ} 30^{\prime}$ gefunden wurde

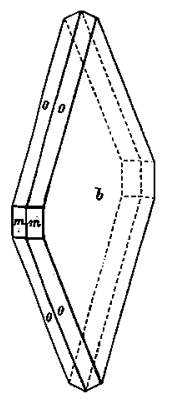

$$
\begin{aligned}
& b: m=(010)(110)={ }^{* 580} 55^{\prime} \\
& b: o=(010)(111)={ }^{6} 69
\end{aligned}
$$

Optische Axenebene (001), erste Mittellinie $b$. Der Axenwinkel in Luft beträgt für $\mathrm{Na}$-Licht

$$
2 E=100^{\circ} 56^{\prime} \text {. }
$$

Die Dispersion ist äusserst gering, $\varrho>v$.

\section{Bibromamidophtalsäureäther.}

$$
\mathrm{C}_{6} \mathrm{HBr}_{2} \mathrm{NH}_{2}\left(\mathrm{CO}_{2} \mathrm{C}_{2} \mathrm{H}_{5}\right)_{2} \text {. }
$$

(F. Wagner, Inaugural-Dissertation, München 1881.)

Krystallsystem mo nosymmetris ch.

$$
\begin{aligned}
a: b: c & =1,4605: 1: 1,4106 \\
\beta & =57052^{\prime} .
\end{aligned}
$$

Schwach röthlich gefärbte, compacte Krystalle von

Fig. 4. starkem Glasglanz. Durch Vorherrschen von $a=(100)$

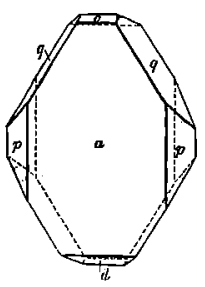
$\infty P \infty$ und $q=(011) R \infty$ meist tafelförmig entwickelt (s. Fig. 4). Die Fläche $a(100)$ ist nach der Längsaxe gestreift. $p=(210) \infty \$ 2, d=(\bar{T} 01) P \infty$ und $c=(001) 0 P$ treten nur untergeordnet auf. Durch das Orthopinakoid

\begin{tabular}{|c|c|c|}
\hline$c: q=(001)(011)$ & $\begin{array}{lll}* 50^{0} & 4^{\prime}\end{array}$ & - \\
\hline$c: a=(001)(100)$ & *57 52 & - \\
\hline$c: d=(001)(\bar{T} 01)$ & *59 16 & \\
\hline$a: q=(100)(011)$ & $70 \quad 10$ & $70^{\circ} \quad 2^{\prime}$ \\
\hline$: p=(100)(210)$ & 3150 & $31 \quad 44$ \\
\hline$p=(011)(210)$ & 4621 & $46 \quad 4 \frac{1}{8}$ \\
\hline$d=(011)(\overline{1} 01)$ & $70 \quad 48$ & 7051 \\
\hline
\end{tabular}
ist keine optische Axe sichtbar. Die beim Schleifen eintretende Trübung der an und für sich schon nicht ganz durchsichligen Krystalle verhinderte eine nähere optische Untersuchung. Spaltbarkeit unvollkommen nach $a(100)$.

Beobachtet: Berechnet:

\section{Picolindicarbonsäure.}

$$
\mathrm{C}_{6} \mathrm{H}_{5} \mathrm{~N}\left(\mathrm{CO}_{2} \mathrm{H}\right)_{2} \text {. }
$$

(W. König s, Ber. d. d. chem. Ges. 1881, 14, 103.)

Krystallsystem rhombis ch.

$$
a: b: c=0,58007: 1: 0,31402 \text {. }
$$


Kleine rechteckige bis $1 \mathrm{~mm}$ dicke Täfelchen, an Fig. 5. denen als schmale Begrenzung des vorzugsweise entwickelten Brachypinakoids $b(010)$ nur noch die Flächen

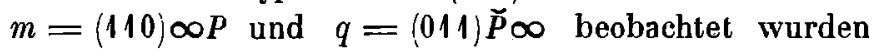
(s. Fig. 5). Durch $b$ keine optischen Axen sichtbar. Spalt-. barkeit nicht wahrnehmbar.

Beobachtet: Berechnet:

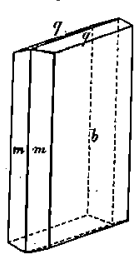

$$
\begin{aligned}
& m: m=(110)(170)={ }^{*} 60^{0} 14^{\prime} \quad \text { - }
\end{aligned}
$$

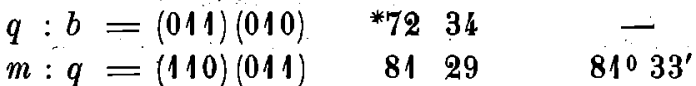

$$
\begin{aligned}
& m: b=(110)(010) \quad 59 \quad 51 \quad 59 \quad 46 \\
& q: q=(011)(0 T 1) \quad 34 \quad 31 \quad 34 \quad 52
\end{aligned}
$$

\section{Nitrochinolin.}

$$
\mathrm{C}_{9} \mathrm{H}_{6} \mathrm{~N}\left(\mathrm{NO}_{2}\right) \text {. }
$$

(W. Kön igs , Ber. d. d. chem. Ges. 1881, 14, 99.)

Dunne, nach der Verticalaxe nadelförmig ausgebildete Prismen ohne Endfläche, welche nach ihrem optischen Verhalten dem mon os ymmet ri s ch e n System angehören.

Die untersuchten Krystalle sind Combinationen der Flächen (100), $(010),(110)$ und $(120)$, sie besitzen starken Glasglanz und bedeutende Dispersion; an einzelnen wurde $Z$ willingsbildung nach dem Orthopinakoid (100) beobachtet.

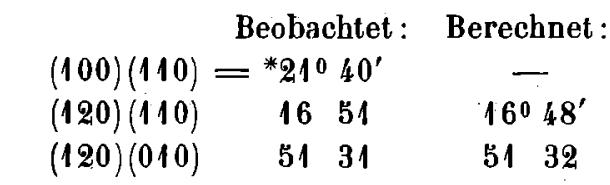

\section{Nitrolepidin.}

$$
\mathrm{C}_{10} \mathrm{H}_{8} \mathrm{~N} .\left(\mathrm{NO}_{2}\right) \text {. Schmelzpunkt 125-1270. }
$$

(W. Kö nigs, Ber. d. d. chem. Ges. 1879, 12, 4.48 und "Studien über die Alkaloide" S. 62.)

Krystallsystem $r \mathrm{hombis} \mathrm{ch}$.

$$
a: b=0,795: 1 \text {. }
$$

Dunne rhombische Tafelchen mit vorherrschender Basis (001) und äusserst schmalen Prismenflächen (110).

$$
(110)(1 \overline{1} 0)=77^{0}
$$

Wegen schlechter Ausbildung der Prismenflächen differiren die Messungen an einzelnen Krystallen bis ùm 1 $\frac{1}{2} 0$. Durch die Basis ist im polarisirten Licbte kein Axenbild sichtbar. 


\section{Dimethyldiphenyltetrazon.}

$$
{ }_{C}^{C_{6} H_{5}}>N-N=N-N<\mathrm{C}_{3} \mathrm{H}_{5}
$$

(E. Fis cher, Liebig's Ann. 190, 67.)

Krystallsystem mon os y m metris ch.

$$
\begin{gathered}
a: b: c=1,7422: 1: ? \\
\beta=85^{\circ} 38^{\prime} .
\end{gathered}
$$

Bräunlichgelbe, durchscheinende bis undurchsichtige compacte Krystalle von dickprismatischem Habitus. Beobachtet wurden nur die Flächen (110) und (001).

$$
\begin{aligned}
& (110)(1 \overline{1} 0)=123^{\circ} 13^{\prime} \\
& (110)(001)=8538
\end{aligned}
$$

Spaltbarkeit nicht wahrnehmbar.

\section{9. $\alpha$-Ditolylpropì่onsäure.}

$$
\mathrm{C}_{17} \mathrm{H}_{18} \mathrm{O}_{2} \text {. }
$$

(C. Böttinger, Ber. d. d. chem. Ges. 1881, 14, 1596.)

Krystallsystem monos y m met ris ch.

$$
\begin{aligned}
a: b: c & =0,9925: 1: 1,5797 \\
\beta & =75^{\circ} 4^{\prime} .
\end{aligned}
$$

Fig. 6.

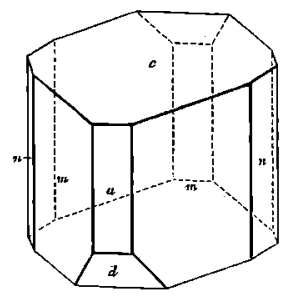

Durchscheinende bis durchsichtige farblose Krystalle, durch fast gleiche Entwicklung von $c=$ (001) $0 P$ und $m=(110) \infty P$ häufig von rhomboëderartigem Habilus. Die Flächen $a=(100) \infty \mathbb{P} \infty$, $d=(10 T) P \infty$ und $n=(120) \infty R 2$ (s. Fig. 6) wurden nur an wenigen Krystallen beobachtet und treten stets nur als schmale Abstumpfungen auf.

Beobachtet: Berechnel:

$$
\begin{array}{llrc}
a: c=(100)(001) & ={ }^{*} 750 & 4^{\prime} & - \\
a: m=(100)(110) & { }^{*} 43 & 48 & - \\
a: d=(100)(10 \overline{1}) & * 35 & 55 & - \\
m: c=(110)(001) & 79 & 29 & 79017 \frac{1}{2}^{\prime} \\
m: d=(110)(10 \overline{1}) & 54 & 27 & 5414 \\
m: n=(110)(120) & 18 & 4 & 1840 \\
a: n=(100)(120) & 6152 & 6228
\end{array}
$$

Optische Axenebene (010), durch $c(001)$ eine Axe sichtbar. Spaltbarkeil nicht vorhanden. 